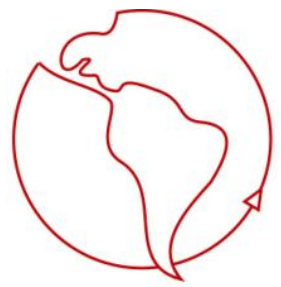

\title{
Capital y Sociedad: Aproximaciones Teóricas y Empíricas
}

Miguel Fernández Llanos

Magíster en Antropología y Desarrollo, Universidad de Chile

babelico@ hotmail.com

\section{Introducción}

En un reciente trabajo se propuso la hipótesis de que el acoplamiento estructural entre la interacción y los sistemas funcionales correspondería a un fenómeno que denominamos capital, que operaría administrando o articulando a los medios de comunicación disponibles en la sociedad y que, gracias a ellos, emergerían repertorios comunicacionales específicos y siempre abiertos a modificaciones que facilitarían una covariación evolutiva propia de los sistemas sociales acoplados (Fernández; 2003).

El presente trabajo ahondará en las reflexiones esbozadas en el texto en referencia, poniendo el acento en el componente empírico que sustenta dichas afirmaciones y en la demarcación precisa de los mecanismos operativos que distinguirían al capital y su rol coordinador aludido.

De acuerdo a lo planteado en dicho artículo (Fernández; 2003) el capital sería un atributo de la sociedad moderna funcionalmente diferenciada que permitiría el acoplamiento estructural entre los sistemas funcionales y los sistemas de interacción. Las razones que permiten sustentar dicha afirmación son básicamente dos: en primer lugar, los sistemas funcionales y la interacción son sistemas sociales autopoiéticos que evidencian estructuras temporales disímiles ya que la interacción tiene un carácter irreversible, mientras que los sistemas funcionales trascienden la irreversibilidad debido a que deben responder a múltiples referencias sistémicas. Así, los sistemas de interacción, dadas ciertas condiciones, pueden sencillamente diluirse y desaparecer tan rápido como aparecieron (Robles; 2002), mientras que los sistemas funcionales mantienen sus comunicaciones orientadas por la prestación, la función y la autorreflexión (Luhmann; 1998: 81-82). En segundo lugar, porque se propone una comprensión con pretensiones de generalidad de los sistemas funcionales, no tanto en lo que ellos son, si no en cómo son observados, presuponiendo que eventualmente los sistemas de interacción son sistemas observadores de su entorno. Si esto último es aceptado, cobra una relevancia especial la afirmación de Luhmann relativa a que los sistemas funcionales, a pesar de su inabarcable diversidad, son comparables (Luhmann; 1998: 148).

El capital como mecanismo coordinador de comunicaciones de órdenes de realidad diversos

Como indicamos, y basados en la formulación primordial de Marx al respecto, el capital es por sobre todo un mecanismo de coordinación, por ende comunicacional, entre los sistemas funcionales y los sistemas de interacción. Básicamente, el capital permite el mantenimiento de la autopoiesis conversacional de la interacción orientándola hacia la tematización de las prestaciones de los sistemas funcionales; por lo mismo, el capital permite simultáneamente la síntesis comunicativa de la prestación, la función y la autorreflexión de los sistemas funcionales, haciendo comunicable esa síntesis para los sistemas en el entorno de los sistemas funcionales. Así en un solo acontecimiento, la operación del capital desata la red de acontecimientos autopoiéticos de ambos tipos de sistemas sociales. De esta forma, se actualizan tipos específicos de comunicaciones, las cuales tienen como soporte a los medios de comunicación simbólicamente generalizados. 
En el caso del sistema funcional de la economía, el medio de comunicación utilizado es el dinero y es a través de él que la interacción vivencia posibilidades de satisfacción de necesidades materiales concretas. En el otro lado de la distinción, a través del capital se generan variaciones positivas de dinero (acumulación) abriendo nuevos abanicos siempre posibles de comunicaciones de carácter económico, es decir, de nuevos negocios en que se reproduce este círculo.

Desde este punto de vista, el capital adquiriría características paradójicas en el sentido de que permite nuevas comunicaciones a través precisamente de la reducción de las posibilidades de comunicación ya que la orientación de las conversaciones de los sistemas de interacción hacia las prestaciones de los sistemas funcionales presupone dicha reducción. En efecto, el capital pone a disposición en su rol coordinador de relaciones intersistémicas lo que distinguimos como repertorios de contingencia $\mathrm{y}$ repertorios de decisión, los cuales permitirán la deriva comunicacional de los sistemas acoplados y corresponden a nuestro entender a lo que Luhmann distinguió como vivencias y acciones, respectivamente (Luhmann; 1998). Estos conceptos aluden a especies de programas de relación intersistémica que reducen la posibilidad de contingencias, entendiendo a ésta como la posibilidad de futuros abiertos que se construyen a partir de lo que sucede en un presente.

Por lo visto, las coordinaciones entre sistemas acoplados manifiestan paradojas que se producen por la inherente actualización de ciertos temas que son amplificadores de la comunicación a través de la armonización de repertorios de contingencia, es decir, de escenarios comunicativos construidos interactivamente, los cuales hacen posible la construcción de repertorios de decisión para así después articular otros escenarios comunicativos en la emergencia y presentación de nuevos repertorios de contingencia. Lo anterior describe un ciclo o mejor dicho una iteración, la cual se restablece permanentemente hasta que se manifiesten las condiciones necesarias para la interrupción de la autopoiesis conversacional de los sistemas de interacción.

Sumado a esto, el hecho de que la interacción tematice las prestaciones de los sistemas funcionales supone una particular sintonía de las múltiples diferencias sistema/entorno que operan en el entorno interno de la sociedad (Luhmann; 1998: 81) ya que los sistemas de interacción vivencian las prestaciones de los sistemas funcionales de manera contextual, es decir, que procesan de manera diferenciada, irreversible e irrepetible la complejidad del entorno. De lo anterior se desprende, y es algo que será muy importante para la reflexión posterior, que los medios de comunicación deben exponer dentro de sus características generalidad (de allí su condición de medios de comunicación generalizados) al mismo tiempo que especificidad. Los medios de comunicación simbólicamente generalizados son al mismo tiempo específicos en el sentido que deben recoger y administrar en la comunicación la contextualidad desatada de la conversación.

¿Cómo es posible que una síntesis selectiva pueda al mismo tiempo mostrar simultáneamente características de generalización y especificidad? Nuestra posición es que no es posible para un medio de comunicación semejante nivel de performatividad. Eso sería posible para los medios de comunicación simbólicamente generalizados si ellos pudieran observar y precisamente esa incapacidad los sitúa en la categoría de medios, no en la categoría de sistemas. Dicho de otra manera, son los sistemas los que en su autopoiesis y en las diferencias que distinguen entre sus respectivas autorreferencia y heterorreferencia los que podrían eventualmente observar la generalidad y la especificidad de los medios de comunicación simbólicamente generalizados a través de distinciones especializadas, las que sin embargo no les permitiría permanente y simultáneamente ejecutar dicha acción observacional. Por lo mismo, lo que hace posible que un medio de comunicación simbólicamente generalizado combine generalización y especificidad, algo que a primera vista aparece como incompatible y por lo tanto imposible, es la presencia de esta forma comunicativa que hemos llamado capital. 
La combinación entre especificidad y generalización supone diferencias entre los medios de comunicación simbólicamente generalizados, lo que redundará en importantes consecuencias para el desarrollo evolutivo del sistema de la sociedad. La relación entre especificidad y generalización que caracteriza a los medios de comunicación simbólicamente generalizados supone una batería de preguntas emergentes relativas a los procesos de construcción social de ellos. Estas preguntas trataremos de esbozarlas en las próximas líneas.

\section{El tránsito de los medios de comunicación simbólicamente generalizados por la red de coordinación capital}

La construcción social de los medios de comunicación simbólicamente generalizados alude a un proceso evolutivo que corre en paralelo a la evolución del sistema de la sociedad y presupone que algunos medios de comunicación evidencian disponibilidades de actualización en la interacción diferenciadas, es decir, que son más factibles de insertarse y participar en los procesos de acoplamiento estructural entre los sistemas de interacción y los sistemas funcionales. En una primera aproximación, el dinero y el poder son medios de comunicación que muestran una presencia más nítida en las comunicaciones del sistema de la sociedad; no implica esto que ellos son necesariamente más importantes que otros medios de comunicación, sino que simplemente participan en la coordinación de una mayor cantidad de procesos comunicativos.

Retomando el lenguaje que da origen a esta discusión, el dinero y el poder evidencian una mayor generalización y especificidad en las relaciones de acoplamiento entre la interacción y los sistemas funcionales porque están disponibles simultáneamente para una multiplicidad de sistemas sociales (generalización) y porque están más habilitados para orientar y encauzar las comunicaciones que se producen indexicalmente en los sistemas de interacción (especificidad).

Por ejemplo, sin lugar a dudas que resulta más recurrente (desde un punto de vista exclusivamente probabilístico) tematizar en la interacción las prestaciones de los sistemas funcionales que involucran al dinero y al poder que a las obras de arte, por ejemplo, ya que esos medios de comunicación posibilitan y al mismo tiempo restringen más procesos comunicativos.

Esto en otras palabras alude a lo que la sociedad en su desarrollo evolutivo le otorga valor, es decir, qué tipo de comunicaciones es relevante tematizar y actualizar y qué tipo de comunicaciones no (Lash; 1997: 20-62). Volveremos sobre este tópico en secciones posteriores de este trabajo.

Esta discriminación entre las comunicaciones que es pertinente tematizar y las que no lo son es la manifestación empírica de la operación del capital. De esta forma, los medios de comunicación transitan a través de la red comunicativa construida por el capital posibilitando complejas y sucesivas articulaciones de repertorios de contingencia y repertorios de decisión. Estos repertorios no corresponden más que a la disponibilidad de diferenciales de medios de comunicación simbólicamente generalizados que son posibles de observar por los sistemas sociales acoplados (sistemas de interacción y funcionales) en el diseño autopoiético y recíproco de sus estrategias comunicacionales. De lo anterior se desprende que una estrategia comunicacional se sustenta en la administración de estas disponibilidades de diferenciales de medios de comunicación, administración que despliega un escenario comunicacional, una particular disposición de acciones y vivencias sistémicas que determinan lo que socialmente es relevante tematizar.

¿Son estas disponibilidades de diferenciales de medios de comunicación atributos de los sistemas sociales? ¿Cómo los sistemas despliegan en un uso específico estos diferenciales? ¿Qué consecuencias tendría el uso de estos diferenciales? Estas preguntas aluden a aspectos extremadamente complejos 
(incluso polémicos) que tienen que ver con la posibilidad de orientar hacia una dirección específica estos escenarios comunicativos. Sin duda que este no es el lugar ni el momento para proponer una respuesta a estas preguntas ya que una respuesta a ellas supondría la emergencia de una racionalidad, es decir, de una direccionalidad orientada por las premisas de una forma de observar específica.

Nuestra posición es que efectivamente existe una posibilidad de que los escenarios comunicativos sean diseñados asimétricamente por los sistemas sociales acoplados, es decir, que debido a disponibilidades diferentes de diferenciales de medios de comunicación simbólicamente generalizados un sistema acoplado pueda proponer posibilidades de selección a otro sistema acoplado. Esto último es perfectamente congruente con la observación teórica referente a la necesaria restrictividad de los procesos comunicativos propios de las sociedades modernas, restrictividad que afecta a la contingencia de los contactos sistémicos.

La asimetría entonces es una condición u ordenamiento estructural de la comunicación y por lo tanto de las sociedades. Esta asimetría en la comunicación en el seno de las sociedades modernas funcionalmente diferenciadas opera a través del capital, ya que éste permite que los medios de comunicación simbólicamente generalizados realicen iteraciones, círculos, los cuales generan acontecimientos autopoiéticos en los sistemas sociales acoplados (Ver Figura 1). Estos círculos, en la medida que se sostienen y generan mayor cantidad de acontecimientos autopoiéticos, producen lo que conocemos (a través de Marx para el caso del sistema funcional económico) como la construcción de una lógica operativa netamente social y que dadas ciertas condiciones describe desde una autorreferencia construida sucesos externos (Luhmann: 1998; 138-139).

Figura 1

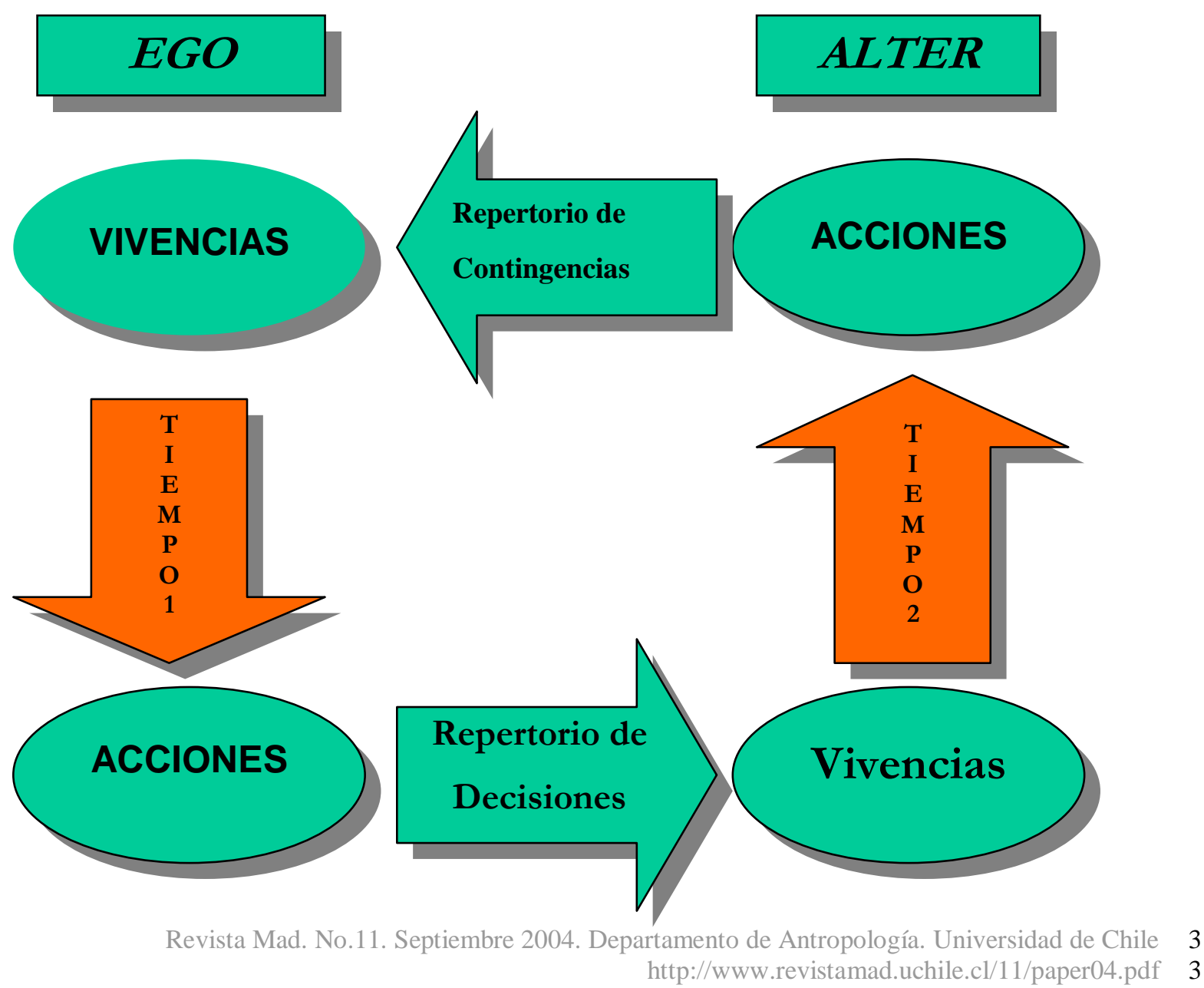


A medida que el proceso de construcción de restricciones recíprocas alcanza espacios y esferas de comunicación nuevas, los sistemas funcionales describen un proceso similar de autodescripción que regula referencias internas y externas, llegando a separarse literalmente de las construcciones de sentido (sociales) que les dieron origen (Luhmann: 1998; 139).

Lo que Luhmann muestra para el sistema funcional económico lo insinúa también para otros sistemas funcionales. Basándose en que todos los sistemas funcionales emergen en un contexto societal y gracias a una particular forma de diferenciación, sugiere que todos los sistemas funcionales son comparables. ¿Qué importancia puede tener la confirmación de esta intuición? Ubicados en nuestro particular punto de vista, y no pudiendo evitar por ahora una simplificación que puede resultar irritante, la importancia implícita en dicha intuición radica en que le permite al análisis sistémico ampliar más todavía sus potencialidades comprensivas, posibilitando a la sociología constituirse en una observadora de la sociedad dotada con herramientas más precisas y asimismo incluyendo en sus descripciones las descripciones de otras disciplinas, incluso externas a las ciencias sociales. Desde allí sugerimos que un punto de comparación posible entre los diversos sistemas funcionales lo constituye el capital, qué medios de comunicación simbólicamente generalizados transitan por el capital específico asociado a cada sistema funcional, qué tipo de restricciones recíprocas produce y, por tanto, qué tipo de repertorios de contingencia y de decisión propone.

\section{Manifestaciones empíricas}

A lo largo de este trabajo hemos intentado argumentar que: Para todo Sistema Funcional existe un Capital $(K)$ tal que $K$ enlaza comunicaciones especializadas con comunicaciones contextualizadas (indexicales), permitiendo el acoplamiento estructural entre los sistemas funcionales y los sistemas de interacción. Dicho enlace se produce gracias a la construcción de repertorios de contingencias y repertorios de decisiones (restricciones recíprocas), los cuales desatan acciones y vivencias sistémicas amplificando así las posibilidades de comunicación. Es imprescindible comprender las acciones y vivencias sistémicas como acontecimientos autopoiéticos (Luhmann; 1995) que tienen sentido y razón en el marco de la unidad de la diferencia sistema/entorno. Dicho de otra manera, los sistemas sociales acoplados a través de estas restricciones recíprocas (repertorios de contingencia y de decisión), presuponen y actualizan autopoiéticamente acontecimientos autopoiéticos que se suscitan en sus respectivos entornos (sin perder su condición de autopoiéticos).

De esto último, que resume sucintamente la aproximación de este trabajo, resalta la fundamental importancia los medios de comunicación simbólicamente generalizados para comprender de una manera más cabal y práctica lo que se quiere mostrar bajo el concepto de capital. Las restricciones recíprocas se pueden diseñar y actualizar en la medida que los sistemas acoplados cuenten con diferenciales de disponibilidad de estos medios de comunicación (dinero, poder, amor, verdad, fe) y en base a las disponibilidades nuevas (actualizadas) de estos diferenciales emergen nuevas comunicaciones que respetan y al mismo tiempo fuerzan un proceso selectivo sustentado en un código binario específico de comunicación (Luhmann; 1998: 104). Descripciones cotidianas (empero extremadamente improbables) como tener/no tener dinero para satisfacer una necesidad o un gusto (algunos piensan que es lo mismo), el verdadero/falso de una proposición científica, la fealdad/belleza de una obra de arte, la salvación/condenación implícita en una determinada actitud religiosa o en un nuevo culto emergente (tan persistente a lo largo de la historia de occidente) o la posibilidad de decidir/no decidir en el ámbito del 
poder político son ejemplos donde se puede observar que las asimetrías en la comunicación operan y despliegan presentes para millones de personas diariamente en el mundo.

La distinción forzosa que plantea la asimetría, cuyo núcleo y génesis lo constituye el capital en el ámbito de la sociedad moderna funcionalmente diferenciada, permite que se construyan interactivamente nuevas posibilidades de disponibilidad de estos denominados diferenciales, permitiendo que se defina socialmente lo valioso en el seno de la sociedad, es decir, que se prioricen decisiones relativas a qué código presente en la sociedad orientará las decisiones que permanentemente deben tomar las interacciones, las organizaciones y, por ende, las sociedades (Lash; op. cit.) (Morandé; 1984). Esto evidentemente remite a las conceptualizaciones que proponen los estudios relativos a la inherencia del riesgo en la sociedad moderna, riesgos que son visibles en la medida en que los sistemas sociales están presionados a tomar decisiones. Sin pretender ser exhaustivos en esta oportunidad, sencillamente porque este es un trabajo que recién comienza y que sin embargo busca la exhaustividad, podemos indicar que emerge un sustento teórico del porqué consideramos al capital en la forma planteada aquí como un atributo distintivo de la sociedad moderna; y es así porque el capital (y su progresión lógica, i.e. restricciones recíprocas, repertorios de contingencia y de decisión y asimetrías en la comunicación) fuerza la necesidad de decisiones en todo ámbito y contexto de la vida social. De esta forma, podemos proponer una tríada de conceptos que desde una perspectiva sociológica distinguen a la sociedad moderna, más allá de la díada fundacional compuesta por la diferenciación funcional y la complejidad, la cual está integrada por las decisiones y el riesgo (Luhmann; 1993, 265) implícito en ellas, cuyo origen, canónico si se quiere, lo encontramos en el capital.

\section{I}

¿Cómo es posible comprender desde una perspectiva sociológica la emergencia de un organismo como la Inquisición? Sin duda alguna que para muchos no sea un ejemplo de lo más adecuado ya que no corresponde a un contexto social marcado por la diferenciación funcional sino por la segmentación. Como respuesta, podemos decir que es una organización dentro de otra organización, la Iglesia, y que debe decidir sobre la presencia de lo prohibido, lo herético, en una, nuevamente, forma diferenciada de experimentación de lo trascendente. En una época en que la concepción global del universo provenía de Dios y por lo tanto de la Iglesia, la Inquisición marca un momento importante en la historia de la diferenciación de la sociedad ya que ella responde a una referencia sistémica que sobrepasaba con creces los ámbitos comunicativos de la organización Iglesia. La Iglesia disponía de los medios de comunicación adecuados para decidir lo que provenía de dios y lo que provenía del demonio, estableciendo claramente para sus fieles (la sociedad de ese tiempo) las posibilidades de distinción entre estos dos entes. Debido a una serie de procesos históricos documentados (Berman; 1992) esa distinción ya inaugurado el primer milenio de era cristiana empieza a opacarse y emergen diversos movimientos de renovación tanto dentro como fuera de la organización Iglesia. Ya que la distinción entre dios y el demonio era crucial para la vida social de esa época, la renovación de las costumbres relativas a la vivencia de esta distinción remeció hasta sus cimientos el adobe social imperante y posibilitó que grupos de personas distintas a la Iglesia se constituyeran como portavoces y portadores de lo trascendente. Si esto hubiera pasado como el desvarío de individuos ciertamente obnubilados por percepciones provenientes del mal no habría habido ningún problema y el establishment se habría mantenido casi sin variaciones, pero la realidad no fue así y miles de personas participaron y vivenciaron la Verdad que tan ajena (desde su perspectiva, obviamente) estaba para los miembros de la Iglesia, desatando una ola de conversión que se extendió por vastas regiones de Europa.

La masividad de dicho fenómeno hace pensar que la ola de renovación espiritual se conectó directamente con demandas concretas, relacionadas con la vida cotidiana de miles de personas y que buscaban un 
ámbito de sentido nuevo y "auténtico". ¿De dónde proviene la búsqueda de esa autenticidad? Desde nuestro punto de vista proviene de la interacción y experimentación directa de los sistemas de interacción con distinciones cuyo contenido de comunicación aludía a experiencias de trascendencia, las cuales se conectaban con condiciones sociales, económicas y culturales muy propicias para la búsqueda de nuevas formas de observación de lo trascendente. De allí que emergieron nuevas organizaciones especializadas en administrar lo trascendente, disputándole decididamente ese rol a los organismos que hasta esa fecha tenían el monopolio de dicha administración.

Aquí viene el aporte interpretativo del capital: la nueva fe desata un sinnúmero de experiencias nuevas que se anclan profundamente en la interacción cara a cara y perfilan nuevos comportamientos (acontecimientos). Esos comportamientos son producidos y posibilitados por una particular distribución del medio de comunicación simbólicamente generalizado fe que, sea cual sea su origen (fascinación, éxtasis, locura), abre expectativas de vivencias (repertorios de contingencia) que distinguen (en ese tiempo) opciones radicales de vida (repertorios de decisión), los cuales fueron considerados inadmisibles e intolerables por los que detentaban también ciertas disponibilidades del medio de comunicación fe.

La tematización de estos nuevos referentes organizacionales de experimentación de lo trascendente por parte de la organización inicial (Iglesia) permitió la andanada de querellas mutuas y que se enmarcaban en las dos posibilidades comunicativas del código herético/no herético. Esas querellas tuvieron una repercusión profunda debido al volumen de ellas, las que dan cuenta de un reconocimiento de parte de una gran masa de personas, articuladas en interacciones sucesivas, de las prescripciones elaboradas por los llamados renovadores y que hicieron posible que estos nuevos referentes de vivencia de lo trascendente fueran considerados como una amenaza para la Iglesia. De allí que la diferenciación interna que da origen a la Inquisición no fuera más que una consecuencia de esta "falla geológica" en la distribución del medio de comunicación simbólicamente generalizado fe en la sociedad de esa época. A través del capital, una vivencia de la fe en la interacción (un acontecimiento autopoiético) desata acciones autopoiéticas en otro sistema social, haciendo que este último condene como equivocadas e intrínsecamente malignas a las primeras, desatándose una ola de violencia y persecución que hasta nuestros días, después de siglos, tiene resonancias: no olvidemos las públicas excusas que ha dado la Iglesia Católica a través de su máxima autoridad por las atrocidades cometidas defendiendo la fe de la paz y el amor. Adelantándonos un poco al segundo ejemplo, no sólo las ideologías presuponen identidad entre valores y estructuras, las organizaciones como la Iglesia también (Morandé; 1984).

Esta reacción violenta se explica en parte, y esbozando una hipótesis, porque los nuevos referentes de experimentación de lo trascendente acumularon, merced a la operación del capital, disponibilidades suficientes de diferenciales de medios de comunicación simbólicamente generalizados como para proponer repertorios de contingencia a los fieles y con ello a los referentes tradicionales de experimentación de lo trascendente, disputándole así la hegemonía (repertorios de decisión) en la administración del medio de comunicación simbólicamente generalizado fe.

\section{II}

¿Cuáles son los antecedentes que explican la crítica hecha por Pedro Morandé al desarrollo de la sociología latinoamericana en general y la sociología chilena en particular? En lo que respecta al valor que le atribuimos al trabajo Cultura y modernización en América Latina no podemos más que decir que se trata del trabajo más sugerente que ha producido la teoría sociológica chilena, a pesar de que 3/4 del libro están dedicados a fundamentar una crítica del quehacer sociológico, al que se le acusa de haber renunciado a todo intento de conceptualizar la realidad propia de nuestras sociedades y de haber desechado una categoría imprescindible para emprender dicha tarea: el ethos cultural latinoamericano. 
Atrapada en un espejismo ideológico como es el desarrollismo, la sociología latinoamericana no puede más que recibir acríticamente las directrices teóricas y metodológicas producidas en Europa o Estados Unidos, con el trasfondo del tránsito de las sociedades latinoamericanas hacia el desarrollo. Este desarrollo obviamente haría más difusa la presencia, en términos de los tipos de distinciones que posibilitaría, de lo que se entiende como la cultura ancestral de nuestros pueblos (Morandé; 1984: 9-127). Dicha omisión, según el autor, condenaría a nuestras sociedades a deambular errantes en medio de la confusión económica y política y a la sociología a automutilarse en medio de marcos teóricos decadentes y menores posibilidades de pensar y describir a la sociedad, tarea que le es propia y determina su razón de ser.

Dejaremos hasta aquí esta minúscula reseña e intentaremos mostrar los mecanismos que operan en esta crítica a través de nuestro concepto estrella capital. Debemos siempre recordar que el capital conecta comunicaciones indexicales provenientes de la interacción con comunicaciones especializadas provenientes de los sistemas funcionales. La labor disciplinar, científica, se construye en nuestros tiempos como redes de conversaciones en que las ideas se reciben, se discuten y se elaboran con ellas programas de investigación, lo quieran o no los científicos involucrados en este proceso.

En medio de esta conversación, los científicos construyen corrientes o tendencias principales que orientan y de alguna manera determinan lo que es relevante de investigar de lo que no. Para que este fenómeno sea observable, la determinación de lo que socialmente es valioso tematizar como práctica científica, debe ser originada por el mecanismo de coordinación capital, ya que en la definición de lo que es relevante investigar para una comunidad científica debe estar presente el componente interaccional. En la interacción se comparten y se institucionalizan prácticas cotidianas que en último término definen lo que es visible para la práctica científica, es decir, como repertorios de contingencia, los cuales desatan acontecimientos autopoiéticos que apuntan a la articulación de repertorios de decisión, cuya manifestación más clara lo constituye, por ejemplo, la definición de las líneas de investigación que son susceptibles de financiar al momento de distribuir los recursos disponibles para dicho fin, ya sea en las universidades, centros de investigación, agencias de cooperación internacional u organismos gubernamentales. El capital, de una u otra manera, intenta responder en este caso particular dos preguntas: ¿Quiénes producen las corrientes principales en materia científica? ¿A través de qué medios?

De esta forma, interactiva e indexicalmente, se construye el marco institucional, político y emocional que orienta la investigación científica en cualquier área del conocimiento humano, situación de la que no escapa actualmente ni escapó en el momento de la crítica de Morandé la sociología chilena y latinoamericana. Este marco institucional, político y emocional corresponde a lo que denominamos las asimetrías en la comunicación, fenómeno que está en permanente tránsito y cambio y que es susceptible de ser negociado si las posibilidades reflexivas de los sistemas sociales acoplados así lo permiten. De esta manera, la investigación científica en sociología describe un desarrollo marcado por múltiples semánticas, ya sea del desarrollismo, culturalismo, marxismo, positivismo, posmodernismo y cualquier otro ismo, pero cuyo mecanismo articulador lo constituye el capital, porque es él el que permite que determinadas comunidades científicas acumulen diferenciales de disponibilidad de medios de comunicación, verdad en este caso.

\section{III}

Ejemplos similares se podrían encontrar en todo el amplio rango de la comunicación social y podríamos pasarnos toda una vida académica argumentando en este sentido. Dejaremos ese ejercicio para una próxima oportunidad, si es que se da. Lo que ahora intentaremos es hacer un ejercicio siempre polémico 
de describir a través de la distinción teórica del capital un futuro posible, el cual como cualquier futuro está siempre disponible.

¿Qué pasaría si un filántropo, magnate y con una paradojal (paradojal desde el punto de vista de las operaciones eminentemente económicas) búsqueda por hacer más equitativa la distribución de un recurso escaso en el Chile de la actualidad, como son las prestaciones de salud de calidad a un precio razonable, se le ocurriera la idea de organizar una Isapre sin fines de lucro? Supongamos que es posible semejante quijotada y que será reconocida desde el sistema de la salud, desde el sistema político y desde el sistema del derecho, permitiendo que esa organización tenga todas y cada una de las obligaciones y derechos de una Isapre orientada hacia la maximización de las utilidades, pero sin este último componente. Es sabido que éste es un negocio con utilidades multimillonarias, por lo tanto la alternativa, que por lo demás es lo que se busca, que se ofrece para las utilidades generadas por esta organización es la inversión en la mejora de sus prestaciones, proceso que devendrá en que esta Isapre hipotética ofrecerá productos y servicios que superará bajo cualquier parámetro de comparación a cualquier otra organización similar. Si sumamos a esto la característica, presente por lo demás en cualquier Isapre, del acceso universal para cualquier cotizante, podemos augurar que la aparición de este tipo de organismo revolucionará desde sus cimientos el mercado de las prestaciones privadas de salud en el país. Esto podrá ser así debido a que una premisa de decisión tan importante como es la posibilidad de lucrar se orienta hacia el cliente y no hacia la organización y su directorio (más a éste último que al conjunto de sus profesionales, técnicos o administrativos). De esta forma, se reorientan radicalmente los que es, nuevamente, valioso en el mercado de la salud privada en Chile, permitiendo que una gran masa de cotizantes prefiera ésta a otra proveedora de servicios de salud.

En este ejercicio se manifiestan al menos dos momentos de operación del capital: en primer lugar en el ámbito de los cotizantes, que verán en esta organización una posibilidad de mejorar sustantivamente un aspecto crucial de su calidad de vida. Eso haría que muchos de ellos prioricen cotizar en esta organización emergente y alocada, asegurando las operaciones de ella, su subsistencia y su desarrollo a través, por ejemplo, de una adecuada remuneración a sus escalafones profesionales y administrativos. En segundo lugar, se desencadenarían procesos comunicativos similares en lo que respecta a esta organización no lucrativa con sus pares lucrativas, ya que la no lucrativa se posicionaría en el mercado como una organización que administra posibilidades de comunicación radicalmente nuevas y diversas, debido fundamentalmente a que despliega de manera diferente el medio de comunicación simbólicamente generalizado dinero en sus relaciones con su entorno interno y externo.

En estos ejemplos, que son una aproximación muy sucinta al trasfondo empírico del concepto, se muestran al mismo tiempo las posibilidades y las dificultades que conlleva la aplicación del concepto de capital en la comprensión de diversos fenómenos sociales que son observables tanto en una problematización estrictamente sociológica como interdisciplinaria. Posibilidades, porque este concepto ofrece una alternativa de explicación concreta y susceptible de ser sometida a examen crítico de fenómenos de carácter masivo y que pueden, dadas ciertas condiciones, cambiar el curso de un gran número de acontecimientos autopoiéticos. Dificultades porque el capital es un concepto teórico y, como vimos, las interacciones y las organizaciones se pueden acoplar simultáneamente a más de un sistema funcional. Entonces ¿Cómo opera el capital acoplando a diversos sistemas que responden a diversas referencias sistémicas y que utilizan diversos medios de comunicación en sus relaciones intersistémicas? Esta pregunta concierne a cómo la operatoria del capital se asocia con los elementos de la operatoria sistémica como sentido, autorreferencia, estructura, doble contingencia, organización y cómo los repertorios de contingencia y de decisión se manifiestan ante ellos, los cuales constituyen, entre otros, la unidad de la diferencia sistema/entorno. En otras palabras, el desafío en lo que viene consistirá en mostrar cómo los repertorios de contingencia y de decisión desatan acontecimientos autopoiéticos en los sistemas 
acoplados; ¿Son esos acontecimientos efectivamente autopoiéticos? Desde nuestro punto de vista sí, ya que como se ha mostrado en otras investigaciones, un sistema social puede ser observador de otro sistema asumiendo (heterorreferencialmente) que su organización sea alopoiética, sin embargo al observar en su unidad autoorganizada se tiene un proceso autopoiético en operación (Hidalgo; 2003).

Como concepto teórico, el capital puede anticipar algunas respuestas a estas preguntas, sin embargo no puede por sí solo referir simultáneamente a todos los acoplamientos estructurales entre sistemas, otorgándole con ello al tiempo una dimensión restrictiva insoslayable. Tiempo y capital van de la mano: desde su formulación más inicial, el capital articula dimensiones temporales en principio divergentes y, siendo un instrumento teórico de observación, no le cabe por el momento más que observar secuencialmente procesos de acoplamiento. Ello no significa que el concepto esté constreñido a ciertos niveles de performatividad determinada; por el contrario, el capital al acentuar la inherente presencia de las decisiones en un acoplamiento, permite que la observación de ellos pueda ser rastreada a partir de las decisiones que desatan estos acoplamientos, específicamente en lo concerniente a qué medios de comunicación son tematizados en esas decisiones y qué procesos desatan la presencia o no presencia de medios de comunicación, poniendo el énfasis por sobre todo en las diferentes disponibilidades (queremos con este término generalizar la noción de cantidad o acumulación) de medios de comunicación simbólicamente generalizados: si la teoría de sistemas hizo tambalear a la racionalidad, también puede hacer tambalear la distinción clara que existe entre medios y fines, reafirmando la centralidad de ambos pero asumiendo que la distinción entre un medio y un fin es una apreciación autorreferente de circunstancias que emergen en la heterorreferencia ¿Aproximaciones a una problematización sociológica de la ética y la moral? Puede ser, por qué no: un rastreo de las decisiones que emergen a partir de un acoplamiento estructural se puede realizar en el marco del concepto de sacrificio (desde el punto de vista utilizado por Morandé), es decir, ¿por qué se decide sacrificar algo si ese algo es lo que le da sentido a una instancia de experimentación social (sistema)? ¿Qué acontecimientos y la presencia de qué medios de comunicación permiten distinguir una solución sacrificial (holocáustica) a un problema social?

\section{Conclusiones}

Hemos argumentado a favor del capital en tres direcciones que convergen en una comprensión teórica que muestra como partícipes de un mismo proceso de constitución de la sociedad a su respectiva diferenciación e integración. Estas tres direcciones configuran una hipótesis abierta de cómo es posible considerar a un flujo de comunicaciones contingentes una sociedad. Contingencia presupone diferencia y sociedad presupone coordinación manteniendo la diferencia: en este sencillo ejercicio lógico sustentamos la afirmación anterior y avizoramos un paso importante que puede dar la sociología en su desarrollo presente y futuro. Esa proposición visionaria está implícita a lo largo de toda la obra de Luhmann y nosotros no hacemos más que transitar por ese camino posible.

Estas tres dimensiones pueden resumirse en que el capital está presente permanentemente en nuestra vida cotidiana, tal como lo afirman Marx y Bourdieu (Bourdieu; 1997). Guardando la distancia necesaria con estas dos figuras, debemos reconocer que sus respectivas aproximaciones pueden constituirse en herramientas muy potentes para consolidar la apertura teórica y la renovación comprensiva que supone la obra de Luhmann para la sociología, de la cual este artículo quiere ser partícipe.

Concretamente, la primera dirección corresponde a que el capital permite modular las comunicaciones de los diversos sistemas sociales que emergen de la diferenciación sistémica, comunicaciones que necesariamente deben ser restringidas para amplificarse. En segundo lugar (tal vez en primer lugar en importancia en la estructura de este artículo), el capital permite que los sistemas sociales en los múltiples acoplamientos en que participan construyan realidades que experimentan otros sistemas y experimenten 
realidades que son construidas por otros sistemas. Lo que distingue a los sistemas funcionales de otros sistemas como las interacciones es que los primeros adquieren una deslocalización y descontextualización, es decir, están disponibles para cualquier lugar espacio-temporal y para cualquier contexto indexical. Esto último creemos que es un avance teórico-metodológico ya que desacopla en forma práctica a los sistemas funcionales de las organizaciones que tematizan también las prestaciones de ellos. En otras palabras (y aunque resulte elemental destacarlo), un banco no es un sistema funcional económico, ni siquiera el FMI; ellas son organizaciones que tematizan en su operar las comunicaciones de los sistemas funcionales. Así, los sistemas funcionales proveen repertorios de decisiones, los cuales se constituyen en largos procesos evolutivos que afectan a la sociedad en su conjunto y que determinan el curso de la distribución de medios de comunicación, siempre escasos, pero siempre posibles de ser si se quiere inventados en ese mismo desarrollo, tal como lo demuestra Norbert Elias en su obra El proceso de la civilización, al fundamentar que las normas de etiqueta distinguen a sus poseedores no sólo porque conocen y utilizan pautas de comportamiento nuevas, sino porque su uso les permitirá a sus usuarios acceder a otros beneficios asociados a la vida cortesana, como la protección de un monarca o una ubicación favorable en la naciente estructura burocrática de los incipientes estado-naciones de la época (Elias; 1993). Algunos objetarán nuevamente que la investigación de Elias se enmarca en un contexto de sociedad segmentada o estratificada y no diferenciada funcionalmente: lo único que puedo responder en este momento ante esas críticas es que la humanidad no se acostó una noche estando segmentada y se levantó estratificada, así como tampoco se acostó una noche estratificada y se despertó, como una víspera de Año Nuevo, diferenciada funcionalmente. Desde que la sociedad es sociedad que se tematizan comunicaciones diferenciadas referidas al poder político, al amor, a la belleza, a la satisfacción de necesidades materiales. Así, los sistemas funcionales, si se me permite la licencia, corresponden más bien a cúmulos de emisiones de comunicaciones que asumen cierto carácter y que restringen o modulan de una forma específica las comunicaciones establecidas con otros sistemas sociales en su entorno.

La tercera dirección mencionada quiere mostrar el trasfondo empírico del concepto, con el fin de poner de manifiesto las posibilidades interpretativas de fenómenos en que se movilizan grandes cantidades de medios de comunicación simbólicamente generalizados, los que identificamos coloquialmente como recursos. Al movilizarse una gran cantidad de medios de comunicación simbólicamente generalizados indica que en vastos sectores de la sociedad está operando el capital coordinando comunicaciones de órdenes de realidad divergentes, proceso que determina la masividad de determinado fenómeno; de manera simplificada podemos establecer una regla en estos términos: a mayor masividad de un fenómeno social $\mathrm{X}$, implica que mayores acoplamientos a través del capital se han suscitado debido a que mayor cantidad de sistemas de interacción se enlazan a comunicaciones específicas provenientes de los sistemas funcionales. Aquí apreciamos otra posible dificultad de esta formulación, relacionada con que los sistemas funcionales son los únicos que permitirían, en su acoplamiento con la interacción, desatar fenómenos de gran alcance y profundidad en el sistema de la sociedad ya que son los únicos que en su autopoiesis articulan una prestación (Luhmann; 1998: 81), sin la cual la operación del capital es imposible (Fernández; 2003).

Como se apreciará, emergen desde esta formulación más preguntas que respuestas, las cuales naturalmente se originan por la naturaleza misma de este programa de investigación ya que las posibilidades interpretativas, si bien se amplifican, también demandan esfuerzos interdisciplinarios cada vez más explícitos e institucionalizados. Sin embargo si asumimos la línea seguida en este trabajo tenemos que pensar como una posibilidad al menos de que las-cosas-pueden-ser-de-otro-modo.

\section{Bibliografía}


Berman, Morris. 1992. Cuerpo y Espíritu. La historia oculta de occidente. Santiago: Editorial Cuatro Vientos.

Elias, Norbert. 1993. El proceso de la civilización. Investigaciones sociogenéticas y psicogenéticas. Buenos Aires: Fondo de Cultura Económica.

Fernández, Miguel. 2003. "Capital, sistemas funcionales e interacción: pasos teóricos tentativos para la descripción de las relaciones de acoplamiento estructural entre sistemas de interacción y sistemas funcionales". Revista MAD, № 9, septiembre.

Hidalgo, Luis Antonio. 2003. "Hacia una Economía Política Global Postmoderna". Cinta de Moebio, N 17, septiembre.

Lash, Scott. 1997. Sociología del posmodernismo. Buenos Aires: Editorial Amorrortu.

Luhmann, Niklas. 1993. Sistemas sociales. Lineamientos para una teoría general. México: Anthropos.

Luhmann, Niklas. 1995. "La autopoiesis de los sistemas sociales”. Zona Abierta 70/71.

Luhmann, Niklas. 1998. Complejidad y modernidad. De la unidad a la diferencia. Madrid: Editorial Trotta.

Morandé, Pedro. 1984. Cultura y modernización en América Latina. Santiago: Cuadernos del Instituto de Sociología. Pontificia Universidad Católica de Chile.

Robles, Fernando. 2002. "Sistemas de interacción, doble contingencia y autopoiesis indexical". Cinta de Moebio, $\mathrm{N}^{\circ} 15$. 